\title{
Non-Invasive Assessment of Vascular System Function and Damage Induced by Anthracycline Treatment in the Pediatric Cancer Survivors
}

\author{
K. BUDINSKAYA ${ }^{1}$, V. PUCHNEROVÁ ${ }^{1}$, J. SVAČINOVÁ ${ }^{1}$, J. NOVÁK $^{1}$, H. HRSTKOVÁ ${ }^{2}$, \\ M. NOVÁKOVÁ ${ }^{1,3}$, A. PEKAŘOVÁ ${ }^{1}$, M. PEKAŘ ${ }^{1}, Z^{\text {. NOVÁKOVÁ }}{ }^{1}$ \\ ${ }^{1}$ Department of Physiology, Faculty of Medicine, Masaryk University, Brno, Czech Republic, \\ ${ }^{2}$ Department of Paediatric Oncology, Faculty of Medicine and Faculty Hospital Brno, Masaryk \\ University, Brno, Czech Republic, ${ }^{3}$ International Clinical Research Center, St. Anne's University \\ Hospital Brno, Brno, Czech Republic
}

Received March 24, 2017

Accepted September 6, 2017

\begin{abstract}
Summary
Anthracyclines represent one of the important classes of anti-cancer drugs; however, their major disadvantage is their profound cardiovascular toxicity. This study aimed to evaluate influence of anthracyclines on cardiovascular stiffness parameters estimated from pulse wave (PW). PW was measured in 59 cancer survivors treated with anthracyclines in childhood and in 248 healthy age-matched controls. Both patients and controls were divided into three age groups $(13-15,16-18$ and 19 - 24 years). Central PW augmentation index $\left(\mathrm{C}-\mathrm{AI}_{75}\right)$ and augmentation pressure $\left(C-A P_{75}\right)$, both normalized to heart rate $75 \mathrm{bpm}$, were calculated as parameters of arterial wall stiffness. Central Buckberg sub-endocardial viability ratio (SEVR) was calculated as a parameter of diastolic function. Patients and controls were compared in each age group. $\mathrm{C}-\mathrm{AI}_{75}$ and $\mathrm{C}-\mathrm{AP}_{75}$ were significantly increased in patients in age groups $16-18$ and 19 - 24 years. SEVR was decreased in patients in the oldest age group. Our results suggest that although toxic influence of anthracyclines to arterial wall and heart are developing during childhood and puberty, they can be detected rather in the adulthood. These changes are yet subclinical; however, their presence indicates potentially increased cardiovascular risk in childhood cancer survivors treated with anthracyclines during childhood.
\end{abstract}

\section{Key words}

Anthracyclines • Cardiotoxicity • Pulse wave velocity • Pulse wave analysis • Arterial stiffness

\section{Corresponding author}

J. Svačinová, Department of Physiology, Faculty of Medicine, Masaryk University, Kamenice 5, 62500 Brno, Czech Republic. E-mail: svacinova@med.muni.cz

\section{Introduction}

Anthracyclines represent one of the important classes of anti-cancer drugs, with major disadvantage in their profound cumulative-dose-dependent cardiovascular toxicity. Despite this fact, they are still widely used in various oncological treatment protocols for both solid tumours and hematological malignancies, such as acute lymphoblastic leukemia, the most frequent type of leukemia in childhood (Elliott 2006, Hortobágyi 1997). Anthracyclines exert their anti-cancer effects by several mechanisms. They inhibit topoisomerase II, enzyme responsible for eliminating supercoiling during DNA replication. They further induce damage of DNA by intercalating into its structure and by promoting the creation of reactive oxygen species (ROS), thus also causing lipid peroxidation (Lipshultz et al. 2008, Puma et al. 2008, Vejpongsa and Yeh 2014).

However, all the above mechanisms affect not only the cancer cells but also other cells within the organism. Apart from well-known damage to myocardium, damage to endothelial cells has been also reported (Chow et al. 2006, Murata et al. 2001, Wu et al. 2002). Anthracyclines cause overexpression of 
inflammatory cytokines in endothelial cells and diminish production of endothelin and nitric oxide (NO) (Chow et al. 2006, Drímal et al. 2006, Duquaine et al. 2003, Lakatta 2003). Therefore, endothelial cells are more susceptible to apoptosis and the functional integrity of endothelium is vitiated (Murata et al. 2001, Wu et al. 2002). The endothelial cells lose their ability to regulate the vascular smooth muscle tone and arterial stiffness increases (Chaosuwannakit et al. 2010, Jenei et al. 2013).

Arterial stiffness is an independent predictor of cardiovascular morbidity and mortality in adult patients who suffer from hypertension or diabetes (Boutouyrie et al. 2002, Laurent et al. 2001). Increased arterial stiffness augments the afterload for left ventricle (LV) which can lead to LV hypertrophy and dysfunction (London et al. 2004, Sutton-Tyrrell et al. 2005).

The damages caused by anthracycline treatment to myocardium and blood vessels might appear right after the anthracycline treatment and are then referred to as the acute cardiotoxicity, However, it can remain silent until adulthood, thus resulting - in the long-term - to chronic or late cardiotoxicity. Predictive factors that would enable identification of patients at the highest risk are still missing and there is therefore the need for the development of novel and sensitive screening modalities enabling detection of subclinical changes and their monitoring (Lipshultz et al. 2013).

The aim of this study was to determine if subclinical changes caused by anthracycline treatment in childhood can be diagnosed by non-invasive measurement of pulse wave velocity (PWV) and aortic pulse wave analysis and to determine when the first subclinical changes can be detected.

\section{Methods}

\section{Subjects}

The present study was approved by Ethics Committee of Masaryk University. It was conducted in accordance with the rules stated in the Declaration of Helsinki.

Altogether, 308 individuals were enrolled to the study. All subjects were informed about the aim and purposes of the study and they signed the informed consent; in patients bellow the age of 18 , the signed consent was obtained from their parents.

Out of 308 enrolled individuals, 59 were childhood cancer survivors (patients; 31 males/ 28 females, age $13-24$ years) and remaining 248 were age-matched healthy controls (139 males/109 females, 13 - 24 years). The patients were treated with anthracyclines during childhood either due to acute lymphoblastic leukemia $(n=34)$ or due to other malignancies such as myeloid leukemia or both Hodgkin and non-Hodgkin lymphomas $(n=25)$. Their anthracycline therapy consisted either from doxorubicin (ADR) or daunorubicin (DNR) in the cumulative dose of 220 (180 - 240) $\mathrm{mg} / \mathrm{m}^{2}$, used together with either cyclofosfamid (cyclo, cumulative dose $3,000(2,800-3,300) \mathrm{mg} / \mathrm{m}^{2}$ ) or vincristine (VCR; cumulative dose $12(12-15) \mathrm{mg} / \mathrm{m}^{2}$ ). Age of the patients at the time of the treatment termination was $4-15$ years. The follow up (i.e. time from the last chemotherapy to the date of cardiovascular system examination) was $4-20$ years. All the descriptive parameters for both patients and control groups are presented in Table 1.

All the individuals in both groups were further divided into three age subgroups $(13-15,16-18$ and $19-24$ years) to reflect the age-dependent changes of cardiovascular variables. Year $\mathrm{N}$ contains patients in interval between $\mathrm{N}^{\text {th }}$ a $(\mathrm{N}+1)^{\text {th }}$ birthdays (day of birthday $(\mathrm{N}+1)$ is excluded from interval).

\section{Data collection}

All the measurements were performed in the quite room with the air temperature of $20^{\circ} \mathrm{C}$. Pulse wave velocity ( $\mathrm{PWV}$ in $\mathrm{m} / \mathrm{s}$ ) was measured by applanation tonometry (Sphygmocor; AtCor Medical, Australia). Measurement was performed on the dominant hand on radial artery and on the ipsilateral carotid artery. PWV was calculated as dividing the distance between carotid and radial arteries by the difference in transition times, i.e. times needed for the pulse wave to transfer from the heart to the site of measurement. Transition times were obtained from the recording as the intervals between the R-wave on the ECG curve and the steep increase on the pulse wave curve either on carotid or radial artery. All the measurements were calibrated to blood pressure that was obtained by oscillometric automated measurement on the brachial artery (Omron HEM-907-E, Japan).

\section{Vascular parameters}

Using the above described applanation tonometry, peripheral pulse wave curve was obtained and systolic (SBP) and diastolic (DBP) blood pressures [mm Hg] were calculated. Central aortic wave was further converted/synthesized using the SphygmoCor Px 
system from the measured peripheral pulse waveform and following parameters were calculated from this central (aortic) curve (as shown in Fig. 1): augmentation pressure (C-AP [mm Hg]; as a difference between the pressure of the first systolic inflection (P2) and the pressure of the systolic peak (P1)) and augmentation index (C-AI [\%], as a percentage expression of the C-AP to the aortic pulse pressure). Because of these parameters are influenced by heart rate, their values were standardized to a heart rate of $75 /$ min and pulse height and in the further text these values will be referred as $\mathrm{C}-\mathrm{AP}_{75}$ and $\mathrm{C}-\mathrm{AI}_{75}$.

Table 1. Basic characteristics of patient and control data set.

\begin{tabular}{|c|c|c|c|}
\hline Patients & & & \\
\hline Age (years) & $13-15$ & $16-18$ & $19-24$ \\
\hline$N($ male/female $)$ & $15(6 / 9)$ & $23(14 / 9)$ & $21(11 / 10)$ \\
\hline$B M I\left(\mathrm{~kg} / \mathrm{m}^{2}\right)$ & $17.8(16.4-20.0)$ & $20.8(17.4-23.7)$ & $23.5(21.8-24.7)$ \\
\hline $\mathrm{SBP}(\mathrm{mm} \mathrm{Hg})$ & $107(100-110)$ & $111(106-127)$ & $114(107-120)$ \\
\hline$D B P(m m ~ H g)$ & $56(59-69)$ & $65(59-68)$ & $66(60-72)$ \\
\hline$H R(b p m)$ & $74(67-80)$ & $67(62-79)$ & $73(69-78)$ \\
\hline follow up (years) & $6.5(5-9)$ & $11(9-13)$ & $14(10-19)$ \\
\hline$n-A D R$ & 6 & 12 & 9 \\
\hline$n-D N R$ & 9 & 11 & 12 \\
\hline \multicolumn{4}{|l|}{ Healthy controls } \\
\hline age (years) & $13-15$ & $16-18$ & $19-24$ \\
\hline$N($ male/female $)$ & $68(39 / 29)$ & $58(21 / 37)$ & $122(79 / 43)$ \\
\hline$B M I\left(\mathrm{~kg} / \mathrm{m}^{2}\right)$ & $19.8(19.3-21.9)$ & $21.1(20.1-22.5)$ & $21.3(19.8-23.1)$ \\
\hline$S B P(m m H g)$ & $110(103-117)$ & $111(102-117)$ & $114(107-120)$ \\
\hline$D B P(m m ~ H g)$ & $61(53-67)$ & $63(59-68)$ & $67(62-71)$ \\
\hline$H R(b p m)$ & $80(75-87)$ & $71(63-78)$ & $68(62-74)$ \\
\hline
\end{tabular}

Parameters are expressed as median (lower quartile - upper quartile) of BMI (body mass index), SBP (systolic blood pressure), DBP (diastolic blood pressure) and HR (heart rate). $\mathrm{N}$ represents number of subjects in each age group. Parameters n-ADR and $\mathrm{n}$-DNR in each group represent number of patients, who used these medicaments.

\section{Parameters of cardiac function}

In order to describe both the systolic and diastolic function of the heart, following parameters were assessed (Fig. 1): central tension time index (the area under the systolic part of the pressure waveform per minute systolic parameter; C-TTI), central diastolic time index (area under the diastolic part of the curve, diastolic parameter; C-DTI), central Buckberg index (the ratio of C-DTI to C-TTI expressed as a percentage that serves as an index of subendocardial viability; SEVR, [\%]).

\section{Statistics}

Data were first tested for the distribution that was shown to be non-Gaussian. Thus, non-parametric analysis was performed and data are expressed as median (lower quartile, upper quartile) for each analyzed parameter. Analyzed cardiovascular variables included:

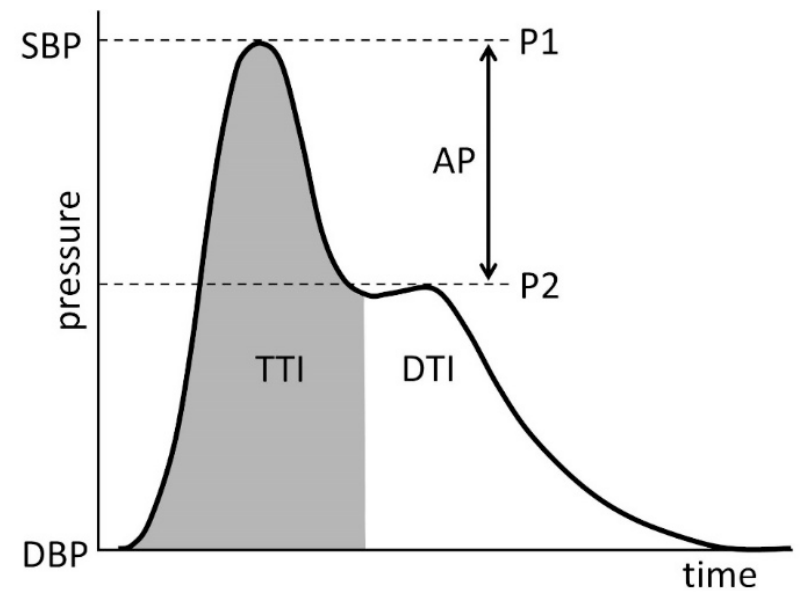

Fig. 1. Pulse pressure waveform. SBP is systolic blood pressure, DBP is diastolic blood pressure. P1 is peak systolic pressure, P2 is pressure of the first systolic inflection. AP is augmented pressure difference between P2 and P1 (AP = P2 - P1). TTI (grey area) is tension time index, DTI (white area) diastolic time index. 
C-TTI, C-DTI, SEVR, C-AI $75, \mathrm{C}-\mathrm{AP}_{75}$, and PWV. Mann-Whitney test was used to evaluate significance of variable difference between patients and controls in each age group. Mann-Whitney test was used to evaluate difference of all cardiovascular variables between the patients using ADR and patients treated with DNR. Spearman correlation coefficients between cardiovascular variables and cumulative dose of $\mathrm{ADR}, \mathrm{DNR}$ or VCR were calculated.

\section{Results}

Statistically significant differences between study groups were observed in some of the studied vascular parameters. The results for $\mathrm{C}-\mathrm{AI}_{75}, \mathrm{C}-\mathrm{AP}_{75}$ and PWV are summarized in Figure 2.

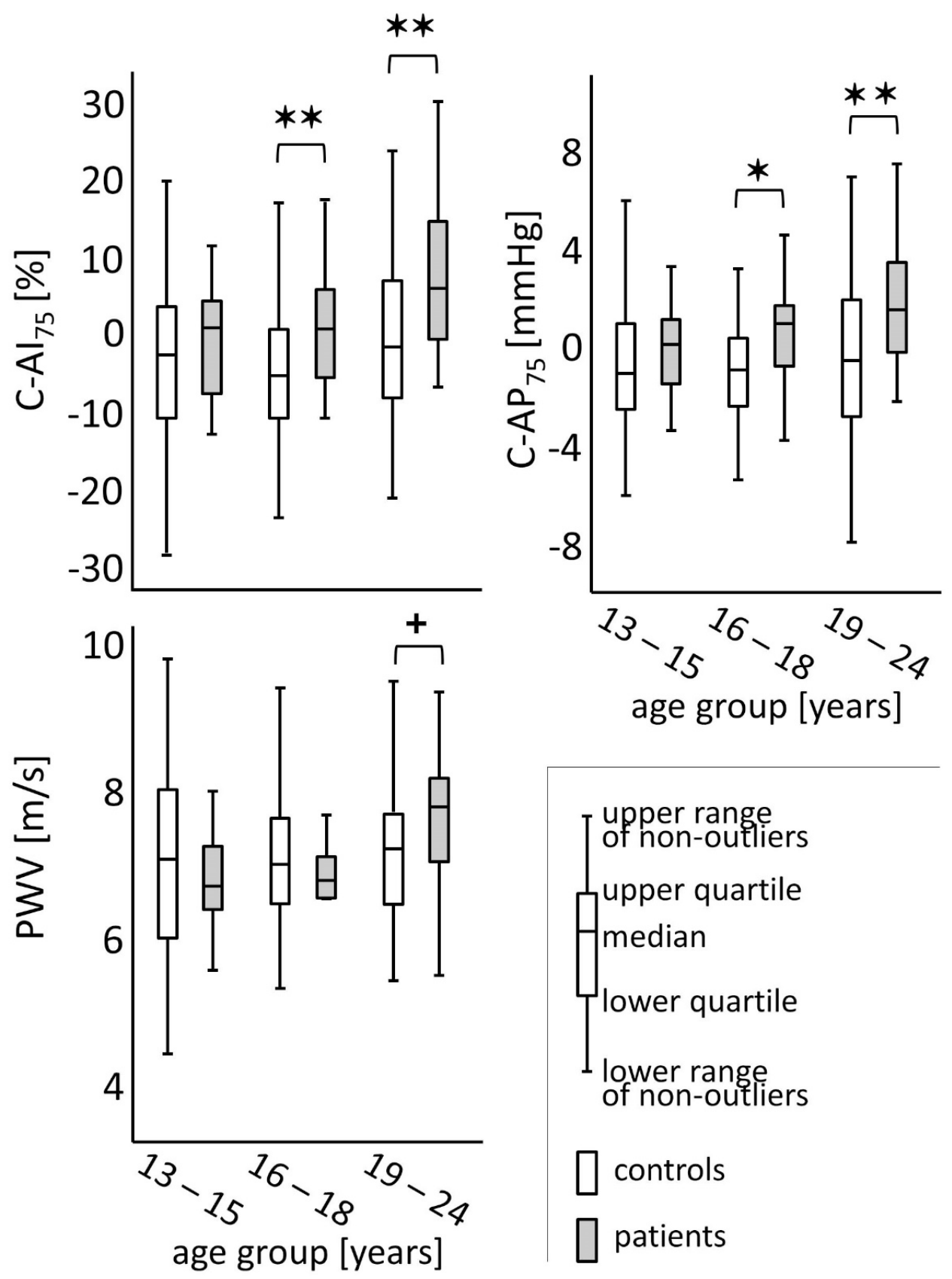

Fig. 2. Distribution (box plots) of vascular parameters: $\mathrm{C}^{-}-\mathrm{P}_{75}$ (central augmentation pressure height normalized on heart rate 75 bpm

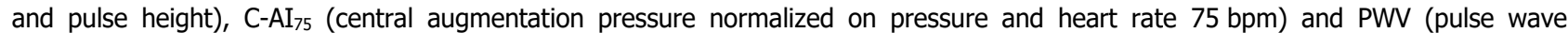
velocity). Significance of differences between patients and controls in each age group (Mann-Whitney test): ${ }^{+} 0.05<p<0.1 ;{ }^{*} p<0.05$; $* * \mathrm{p}<0.01$. 
Corrected parameters $\mathrm{C}-\mathrm{AI}_{75}$ and $\mathrm{C}-\mathrm{AP}_{75}$ were significantly increased in patients than in controls in the older age groups, i.e. in groups $16-18$ years and $19-24$ years. PWV did not differ between patients and controls in younger age groups, however, it was borderline increased $(\mathrm{p}=0.056)$ in the oldest patients' group (19-24 years).

No differences of cardiovascular variables were observed between patients treated with DNR as compared to those treated with ADR. Cardiovascular variables did not correlate with cumulative dose of ADR, DNR or VCR.
Furthermore, statistically significant differences in systolic and diastolic dysfunction variables (C-TTI, C-DTI and SEVR) were also observed (Table 2). C-TTI was significantly increased and SEVR was significantly decreased in patients as compared to controls; however, this was observed only in the age group $19-24$ years. There were no significant differences for C-TTI and SEVR in other age groups and no statistically significant changes for C-DTI in all age groups.

There were no significant differences in the generally used markers (BMI, SBP, DBP, and HR) between patients and controls in all age groups.

Table 2. Medians (lower - upper quartile) of parameters describing cardiac dysfunction.

\begin{tabular}{llccc}
\hline & Age (years) & $\mathbf{1 3}-\mathbf{1 5}$ & $\mathbf{1 6}-\mathbf{1 8}$ & $\mathbf{1 9 - 2 4}$ \\
\hline \multirow{2}{*}{ C-TTI } & controls & $1,691(1,556-2,008)$ & $1,673(1,508-1,855)$ & $\mathbf{1 , 7 4 7}(\mathbf{1 , 5 9 3}-\mathbf{1 , 9 2 9})$ \\
& patients & $1,601(1,540-1,977)$ & $1,725(1,614-1,854)$ & $\mathbf{1 , 8 9 2 *}(\mathbf{1 , 7 5 7 - 2 , 0 8 1 )}$ \\
\hline \multirow{2}{*}{ C-DTI } & controls & $2,708(2,485-3,066)$ & $2,914(2,780-3,194)$ & $3,076(2,882-3,347)$ \\
& patients & $2,963(2,708-3,145)$ & $3,081(2,812-3,219)$ & $3,074(2,807-3,175)$ \\
\hline \multirow{2}{*}{ SEVR } & controls & $153(139-178)$ & $177(158-199)$ & $\mathbf{1 7 7}(\mathbf{1 5 9}-\mathbf{1 9 8})$ \\
& patients & $170(142-201)$ & $173(148-203)$ & $\mathbf{1 6 0 * *}(\mathbf{1 4 6}-\mathbf{1 7 5})$ \\
\hline
\end{tabular}

C-TTI (central tension time index, systolic dysfunction parameter), C-DTI (central diastolic time index, diastolic dysfunction parameter), SEVR (central Buckberg sub-endocardial viability ratio, diastolic dysfunction parameter, \%). Significant difference between controls and patients (Mann-Whitney test): * $\mathrm{p}<0.05 ; * * \mathrm{p}<0.01$.

\section{Discussion}

Anthracycline therapy is known for and is limited by its profound cardiotoxicity. Children cancer survivors are generally acknowledged to be at increased risk for the development of cardiovascular disease (such as coronary artery disease, heart failure or cerebrovascular disease) and are more prone to cardiovascular death (Elliott 2006, Hortobágyi 1997, Oeffinger and Hudson 2004). However, increased cardiovascular risk is probably not mediated only by the direct toxic effects on the heart, but also by the effects of anthracyclines on the vascular system (Chow et al. 2006). Furthermore, changes in the arterial stiffness are known to be related to increased cardiovascular risk (Boutouyrie et al. 2002, Cruickshank et al. 2002). Thus, within this study, childhood-cancer survivors vascular function was assessed non-invasively using various parameters derived from the peripheral pulse wave curve. Several subclinical differences, especially in the older patients' groups were observed. Our study is in accordance with other authors' finding that were focusing on the arterial stiffness parameters in patients. Several studies in this field have already been conducted; however, as there were various methods for the vascular stiffness and functions assessment used and the significant diversity in the patients' selection was present, studies are not easily comparable.

One of the first clinical studies focusing on arterial stiffness assessment was performed in 2006 on a relatively small sample $(n=14)$ of childhood cancer survivors. Still it showed that brachial artery reactivity (BAR; also known as flow mediated dilation - FMD) is decreased $(6.7 \pm 3.3 \%$ in patients vs. $3.8 \pm 3.4 \%$ in controls) in patients treated with the anthracyclines at the cumulative dose higher than $300 \mathrm{mg} / \mathrm{m}^{2}$ in the age $14.5 \pm 4.54$ years (Chow et al. 2006). Decrease in FMD was later confirmed in larger study $(\mathrm{n}=96)$ by Jenei et al. (2013) $(7.12 \pm 6.28 \%$ in patients vs. $13.13 \pm 2.40 \%$ in controls) in patients with cumulative dose below $350 \mathrm{mg} / \mathrm{m}^{2}$ with the age $13.7 \pm 4.9$ years. Moreover, Jenei et al. (2013) also showed that aortic stiffness index 
determined by echocardiography is also decreased in childhood cancer survivors. Both studies are in accordance with our results showing, that subclinical endothelial dysfunction may be one of the factors contributing to the increased cardiovascular risk in childhood cancer survivors.

Except of BAR/FMD, subclinical atherosclerosis may be detected using the parameters derived from the pulse waveform or using PWV, both of which also reflect endothelial damage and vascular stiffness. Moreover, increased PWV is known as independent predictor of increased cardiovascular morbidity and mortality (Boutouyrie et al. 2002, Cruickshank et al. 2002, London et al. 2004). Previous studies focusing on the PWV observed statistically significant increase in aortic PWV $(6.24 \pm 1.34 \mathrm{~m} / \mathrm{s}$ in patients vs. $5.42 \pm 0.69 \mathrm{~m} / \mathrm{s}$ in controls, $\mathrm{p}<0.001$ ) assessed by Arteriograph (Herceg-Cavrak et al. $2011)$ in children with the mean age $13.59 \pm 4.44$ years or in carotid-femoral PWV $(6.37 \pm 0.89 \mathrm{~m} / \mathrm{s}$ in patients vs. $5.76 \pm 0.88 \mathrm{~m} / \mathrm{s}$ in controls, $\mathrm{p}=0.012$ ), however, not in children but only in young adults above 18 years (Krystal et al. 2015). Our study showed increase in PWV of borderline significance in older patients, which is in accordance with the previous studies. Study by Herceg-Cavrak involved slightly younger control group (patients $13.59 \pm 4.44$ vs. controls $12.21 \pm 3.0$ years, $\mathrm{p}=0.081$ ) and did not distinguish the age subgroups (Herceg-Cavrak et al. 2011). Since PWV is known to increase with age, these may be the reasons for the differences in the significance between our results. Krystal et al. (2015) assessed PWV by different means and similarly to our study showed that only in older patients, PWV changes are detectable. These results thus suggest that vascular stiffness changes need certain time to develop and as the patients grow older, these changes become apparent. Moreover, some authors found no correlation between PWV and the cumulative dose of anthracyclines (Chaosuwannakit et al. 2010, HercegCavrak et al. 2011, Krystal et al. 2015); on the contrary, Jenei et al. (2013) observed independent association of FMD and aortic stiffness index assessed by echocardiography with anthracycline dose. It can thus be hypothesized that the endothelial dysfunction at its early stages detected by FMD may be related to anthracycline dose; however, more robust changes resulting in increased PWV are dose-independent.

Lastly, to our best knowledge, this is the first study to report changes in the parameters derived from the pulse waveform (both markers of vascular function
C-AI and C-AP and parameters of diastolic and systolic dysfunction SEVR and C-TTI, respectively) in the childhood cancer survivor cohort of this size focusing also on age subgroups. C-AI values were increased in the older age groups (Fig. 2). Increased C-AI is known to be related to increased cardiovascular mortality and is associated with higher target organ damage (Shimizu and Kario 2008). At the same time it is known, that C-AI levels better reflect the response of the organism to vasoactive drugs (such as beta-blockers, ACE inhibitors or AT1-receptor blockers) than the values of PWV (Boutouyrie et al. 2010). Our results are thus indicative, that C-AI may serve as better indicator of vascular damage induced by anthracyclines as compared to PWV and that changes to the vasculature are developing with age, as most of the observed differences were statistically significant only in the older age groups. Concerning the parameters of diastolic dysfunction, SEVR reflects the subendocardial viability which was decreased in the oldest patients' group, while C-TTI reflecting the tension time during systole was increased - both values are reflecting the initial subclinical changes in the diastolic function and if confirmed by further studies, they may be used in the future to detect the patients at risk of developing clinically apparent diastolic dysfunction. There is a question, if the detected changes in vascular stiffness are resulting just form the toxic effect of anthracycline or if they are caused rather by the complex oncologic therapy. This is difficult to answer because all of the patients get a complex therapy, not only anthracycline antibiotics. The toxic effect of anthracyclines to endothelial cells has been already proved by many authors (Jang et al. 2013, Jenei et al. 2013). However, patients treated with cyclophosphamide or vincristine also experience vascular complications (MI, stroke, hepatic veno-occlusion in cyclophosphamide-treated patients and myocardial ischemia and infarction in vincristine-treated patients) (Cameron et al. 2016). Therefore, we can't exclude the influence of other anticancer drugs used in the therapy of our patients. Nevertheless there is strong evidence of the vascular toxicity of anthracycline antibiotics and their influence is indisputable.

The results of the present study show that with ageing of the childhood cancer survivors, the damage caused by the anticancer treatment in the childhood is becoming more apparent as reflected by increased values of several variables, especially in older age groups. This study thus provides rationale for the need of complex 
cardiovascular follow-up of childhood cancer survivors that should include not only regular echocardiography, but also regular assessment of their vascular function. Further studies with longer ("life-time") follow-up are currently needed to determine the best method for vascular impairment assessment and to see, whether the detected subclinical changes will be useful in the prediction of future cardiovascular events.

\section{Conflict of Interest}

There is no conflict of interest.

\section{Acknowledgements}

The work was supported by grant project: MUNI/A/1355/2016, the project co-financed from EU sources and project No. LQ1605 from the National Program of Sustainability II (MEYS CR). The authors wish to thank Eva Zavodna, M.D., Ph.D. and Monika Reznickova, MSc. for their help with obtaining the data.

\section{References}

BOUTOUYRIE P, ACHOUBA A, TRUNET P, LAURENT S: Amlodipine-valsartan combination decreases central systolic blood pressure more effectively than the amlodipine-atenolol combination: the EXPLOR study. Hypertension 55: 1314-1322, 2010.

BOUTOUYRIE P, TROPEANO AI, ASMAR R, GAUTIER I, BENETOS A, LACOLLEY P, LAURENT S: Aortic stiffness is an independent predictor of primary coronary events in hypertensive patients: a longitudinal study. Hypertension 39: 10-15, 2002.

CAMERON AC, TOUYZ RM, LANG NN: Vascular complications of cancer chemotherapy. Can J Cardiol 32: $852-862,2016$.

CHAOSUWANNAKIT N, D’AGOSTINO R, HAMILTON CA, LANE KS, NTIM WO, LAWRENCE J, MELIN SA, ELLIS LR, TORTI FM, LITTLE WC, HUNDLEY WG: Aortic stiffness increases upon receipt of anthracycline chemotherapy. J Clin Oncol 28: 166-172, 2010.

CHOW AY, CHIN C, DAHL G, ROSENTHAL DN: Anthracyclines cause endothelial injury in pediatric cancer patients: a pilot study. $J$ Clin Oncol 24: 925-928, 2006.

CRUICKSHANK K, RISTE L, ANDERSON SG, WRIGHT JS, DUNN G, GOSLING RG: Aortic pulse-wave velocity and its relationship to mortality in diabetes and glucose intolerance: an integrated index of vascular function? Circulation 106: 2085-2090, 2002.

DRÍMAL J, ZÚROVÁ-NEDELCEVOVÁ J, KNEZL V, SOTNÍKOVÁ R, NAVAROVÁ J: Cardiovascular toxicity of the first line cancer chemotherapeutic agents: doxorubicin, cyclophosphamide, streptozotocin and bevacizumab. Neuro Endocrinol Lett 27 (Suppl 2): 176-179, 2006.

DUQUAINE D, HIRSCH GA, CHAKRABARTI A, HAN Z, KEHRER C, BROOK R, JOSEPH J, SCHOTT A, KALYANARAMAN B, VASQUEZ-VIVAR J, RAJAGOPALAN S: Rapid-onset endothelial dysfunction with adriamycin: evidence for a dysfunctional nitric oxide synthase. Vasc Med Lond Engl 8: 101-107, 2003.

ELLIOTT P: Pathogenesis of cardiotoxicity induced by anthracyclines. Semin Oncol 33 (3 Suppl 8): S2-S7, 2006.

HERCEG-CAVRAK V, AHEL V, BATINICA M, MATEC L, KARDOS D: Increased arterial stiffness in children treated with anthracyclines for malignant disease. Coll Antropol 35: 389-395, 2011.

HORTOBÁGYI GN: Anthracyclines in the treatment of cancer. An overview. Drugs 54 (Suppl 4): 1-7, 1997.

JANG WJ, CHOI DY, JEON IS: Vascular endothelial dysfunction after anthracyclines treatment in children with acute lymphoblastic leukemia. Korean J Pediatr 56: 130-134, 2013.

JENEI Z, BÁRDI E, MAGYAR MT, HORVÁTH A, PARAGH G, KISS C: Anthracycline causes impaired vascular endothelial function and aortic stiffness in long term survivors of childhood cancer. Pathol Oncol Res 19: 375-383, 2013.

KRYSTAL JI, REPPUCCI M, MAYR T, FISH JD, SETHNA C: Arterial stiffness in childhood cancer survivors. Pediatr Blood Cancer 62: 1832-1837, 2015.

LAKATTA EG: Arterial and cardiac aging: major shareholders in cardiovascular disease enterprises: Part III: cellular and molecular clues to heart and arterial aging. Circulation 107: 490-497, 2003. 
LAURENT S, BOUTOUYRIE P, ASMAR R, GAUTIER I, LALOUX B, GUIZE L, DUCIMETIERE P, BENETOS A: Aortic stiffness is an independent predictor of all-cause and cardiovascular mortality in hypertensive patients. Hypertension 37: 1236-1241, 2001.

LIPSHULTZ SE, ALVAREZ JA, SCULLY RE: Anthracycline associated cardiotoxicity in survivors of childhood cancer. Heart Br Card Soc 94: 525-533, 2008.

LIPSHULTZ SE, FRANCO VI, COCHRAN TR: Cardiotoxicity in childhood cancer survivors: A problem with long-term consequences in need of early detection and prevention. Pediatr Blood Cancer 60: 1395-1396, 2013.

LONDON GM, MARCHAIS SJ, GUERIN AP, PANNIER B: Arterial stiffness: pathophysiology and clinical impact. Clin Exp Hypertens 26: 689-699, 2004.

MURATA T, YAMAWAKI H, YOSHIMOTO R, HORI M, SATO K, OZAKI H, KARAKI H: Chronic effect of doxorubicin on vascular endothelium assessed by organ culture study. Life Sci 69: 2685-2695, 2001.

OEFFINGER KC, HUDSON MM: Long-term complications following childhood and adolescent cancer: foundations for providing risk-based health care for survivors. CA Cancer J Clin 54: 208-236, 2004.

PUMA N, RUGGIERO A, RIDOLA V, MAURIZI P, LAZZARESCHI I, ATTINÀ G, MASTRANGELO S, DE ROSA G, RICCARDI R: Anthracycline-related cardiotoxicity: risk factors and therapeutic options in childhood cancers. Signa Vitae 3: 30-34, 2008.

SHIMIZU M, KARIO K: Role of the augmentation index in hypertension. Ther Adv Cardiovasc Dis 2: 25-35, 2008.

SUTTON-TYRRELL K, NAJJAR SS, BOUDREAU RM, VENKITACHALAM L, KUPELIAN V, SIMONSICK EM, HAVLIK R, LAKATTA EG, SPURGEON H, KRITCHEVSKY S, ET AL.: Elevated aortic pulse wave velocity, a marker of arterial stiffness, predicts cardiovascular events in well-functioning older adults. Circulation 111: 3384-3390, 2005.

VEJPONGSA P, YEH ET: Prevention of anthracycline-induced cardiotoxicity: challenges and opportunities. J Am Coll Cardiol 64: 938-945, 2014.

WU S, KO YS, TENG MS, KO YL, HSU LA, HSUEH C, CHOU YY, LIEW CC, LEE YS: Adriamycin-induced cardiomyocyte and endothelial cell apoptosis: in vitro and in vivo studies. J Mol Cell Cardiol 34: 1595-1607, 2002. 